\title{
Forward into the second century of Haematologica
}

\author{
Jacob M. Rowe
}

Department of Hematology, Shaare Zedek Medical Center, Jerusalem, Israel

E-mail: JACOB M.ROWE - rowe@rambam.health.gov.il

doi:10.3324/haematol.2020.271767

I

t is a privileged, but humbling, experience to assume the role of Editor-in-Chief of the oldest hematology journal in the world. Haematologica was first published a hundred years ago in January 1920, and since the initial foundations laid down by Adolfo Ferrata and Carlo Moreschi there has been a succession of distinguished editors and deputy editors who have taken the Journal through different generations in the advancement of the science and clinical practice of hematology. Since the Journal was first published in English in 1974, a line of distinguished editors have moved the journal through to its current position at the forefront of its field; these include Edoardo Storti, Edoardo Ascari, Mario Cazzola, Robin Foà, Jan Cools and Luca Malcovati. I am particularly grateful to Luca for the high scientific standards he has set in place for the Journal and for personally helping me prepare for my new role during these months of transition. In addition, I could certainly not do this without the guidance of Carlo Balduini, the Chairman of the Board of the Ferrata Storti Foundation who is also the Deputy Editor of the Journal, and who has spent countless hours educating me on both the exalted history of the Journal and its current thrust and focus.

Haematologica will continue to be a leading journal in the field of hematology and will strive to further the excellence of its publications. The Journal's first priority is to publish high quality studies that are novel and that will have a high impact in the international medical community. We will publish in the area of clinical practice and will continue to focus on randomized and non-randomized trials. Observational studies and meta-analyses will be considered, but the priority will always be for prospectively-designed studies that are likely to impact on current practice. In the area of basic research, we will encourage a focus on genomic and other advanced biological research areas that will evolve from the basic science to the translational areas. All articles submitted will undergo rigorous peer review designed to ensure the best papers are selected. As with all leading hematology journals, where a rigorous prioritization is in place, some very good papers cannot be published. In addition to regular articles, the Journal will also publish review articles, editorials and perspectives; these will usually be written on invitation but individual submissions can also be considered. Letters to Haematologica are high quality papers that will undergo the same standard of peer review as other types of submissions. Letters describe a novel or preliminary finding that is of major interest but not mature enough to constitute a regular article.

As I look forward to embarking on this new adventure, I am joined in partnership firstly by Jerald Radich who will be the Deputy Editor and by a very dynamic group of associate editors who will do the bulk of the peer review assignments and assessments. New members of the Editorial Board will be selected and it is hoped they will take an active part in reviewing papers and exploring new areas in which to solicit publications.

Haematologica remains committed to a rapid review procedure and we are determined to further improve this. The new website was introduced in September and is designed to facilitate the submission of articles as well as providing clear guidelines to authors. Readers are invited to experience these new features, and suggestions and observations are always welcome.

Since the recent worldwide onset of COVID-19, no area of our lives has escaped with impunity. The pandemic has affected authors, reviewers and editors alike. This, along with the proliferation of new academic outlets in hematology, both in print and digital, has presented unique challenges. We at Haematologica are committed to face these. Although primarily an on-line journal, print versions are available to libraries and for discerning individuals. The artistic cover pages from the Haematologica Atlas will continue to grace many of the issues.

The mission that I and my colleagues face is a daunting one, but one that we will meet head-on with enthusiasm, albeit with some trepidation. With quiet confidence, together we can meet the challenge.

Jacob $M$. Rowe

Jerusalem, Israel 